International Journal of Distributed and Parallel Systems (IJDPS) Vol.2, No.3, May 2011

\title{
SOCP APPROACH FOR REDUCING PAPR FOR MIMO-OFDM VIA TONE RESERVATION
}

\author{
Emna Hajlaoui ${ }^{1}$ and Mahmoud Abdellaoui ${ }^{1,2}$ \\ ${ }^{1}$ National Engineering School of Sfax, Sfax University, Tunisia, \\ emhajlaoui@yahoo.fr \\ ${ }^{2}$ National Institute of Electronics and Communications, Sfax University, Tunisia \\ mahmoud.abdellaoui@gmail.com
}

\begin{abstract}
A combination of multiple-input multiple-output with orthogonal frequency division multiplexing has become a promising candidate for wireless communications. However, like OFDM, a serious disadvantage of the MIMO-OFDM system is the high Peak to Average Power Ratio (PAPR), which may severely affected the power efficiency of RF power amplifiers. In this paper, we proposed a novel method to reduce the PAPR based on the use of unused subcarriers. Accordingly, we formulated the reduction of the PAPR problem as space-time block codes approach (convex optimization). The reduction method is originally based on a particular case of Tone Reservation approach which uses the unused carriers of standards. Indeed, this approach does not degrade the bit-error-rate or the data rate and no side information is required. Besides, our approach is relatively simple, robust and precise and it achieved significant reduction in PAPR. In fact, we validated our approach on the WiMax systems and we compared our results with those found in the literature. Consequently, our proposed approach improved a PAPR reduction about $5 \mathrm{~dB}$ at the $10^{-3}$ probability level. In addition, and precisely when using CCDF of signal technique optimized with constraint mean power vs. $\gamma$, the PAPR was reduced about $6 d B$. However and with previously works, a PAPR reduction has been obtained about $2.5 \mathrm{~dB}$.
\end{abstract}

\section{KEYWORDS}

Multiple Input Multiple Output -Orthogonal Frequency Division Multiplexing (MIMO-OFDM), Peak to Average Power Ratio (PAPR), Tone Reservation (TR), Second Order Cone Programming (SOCP).

\section{INTRODUCTION}

MIMO radio systems have attracted considerable interest and have been employed intensively due to its potential of achieving high data rates in multipath channel [1]. It is shown that when the multiple transmit and receive antennas are used to form a MIMO system, the system capacity can be improved by a factor of the minimum number of the transmit and receive antennas compared to a single-input single-output (SISO) system with flat Rayleigh fading or narrowband channels [2]. For high data rate wireless wideband applications, MIMO combined with OFDM is being considered in a large number of current technology applications such as in IEEE $802.16 \mathrm{~d} / \mathrm{e}$ WiMax [3]. Indeed, MIMO-OFDM has attracted much attention because it can realize higher bit-rate and more reliable transmission. MIMO-OFDM can achieve excellent transmission performance over multipath fading channels by the OFDM technique, and can realize space division multiplexing (SDM) using multiple transmit and receive antennas. While OFDM has a great advantage of having simple equalization, it has an inherent drawback of PAPR [4]. In fact, the peak power of a signal is a critical design factor for band limited communication systems, and it is necessary to reduce it as much as possible.

On the other hand, the PAPR limits seriously the power efficiency of the transmitter's High Power Amplifier (HPA). Thus, many techniques have been proposed to deal with the PAPR problem [5-11]. These techniques include amplitude clipping, clipping and filtering, coding, tone reservation, tone injection, active constellation extension, and multiple signal 
representation techniques such as partial transmit sequence, selected mapping, and interleaving. These techniques achieve PAPR reductions at the expense of transmit signal power increase, bit error rate increase, data rate loss and computational complexity increase.

In similar fashion to SISO systems, MIMO-OFDM also suffers from the PAPR problem. Some recent works have investigated MIMO-OFDM PAPR reduction via selective mapping [12], cross-antenna rotation and inversion [13], unitary rotation [14], polyphase interleaving and inversion [15] and optimal PAPR reduction [16].

In this paper, we proposed a general approach of MIMO-OFDM SOCP for reducing PAPR combining the SOCP SISO-OFDM and $2 \times 2$ MIMO-OFDM solutions [17], [18]. Indeed, the objective is to improve the reduction of the PAPR without any degradation of the BER [19] or the data rate and without sending any Side Information. To do so, we will add a generated corrective signal to the unused carriers of the standard.

This paper is organized as follows. After having introduced work, sections 2 described respectively the MIMO-OFDM system and the proposed approach. In section 3, we presented our simulation results for IEEE 802.16d WiMax standard. Finally, section 4 summarized and concluded the paper.

\section{SYSTEM DESCRIPTION AND PROPOSED APPROACH}

At first, we presented the basic PAPR notions and OFDM signal characteristics that we need thereafter. In fact, in an OFDM modulation, we consider a block of $\mathrm{N}$ data symbols $\mathrm{X}_{\mathrm{k}}(k=$ $0,1 \ldots N-1)$, gathered in an $\mathrm{X}$ vectors, and then will be transmitted in parallel such that each symbol $\left(X_{k}\right)$ modulates a subcarrier $f_{k}(k=0,1 \ldots N-1)$. The $N$ subcarriers are orthogonal, with $f_{k}=k \Delta f$, where $\Delta f=1 / T$ and $T$ is the original symbol period. The resulting analog OFDM signal $x(t)$ can be expressed as:

$$
x(t)=\frac{1}{\sqrt{N}} \sum_{k=0}^{N-1} X_{k} e^{j 2 \pi f_{k} t}, t \in[0, T]
$$

In real implementation, the digital transmission signal may be generated by the Inverse Fast Fourier Transform (IFFT) in the transmitter and restored by Fast Fourier Transform (FFT) in the receiver. The PAPR of the OFDM signal can be defined as:

$$
P A P R_{x}=\frac{\max _{t \in[0, T]}|x(t)|^{2}}{E\left\{|x(t)|^{2}\right\}}
$$

Where $x(t)$ is the time domain signal, $T$ is the OFDM symbol duration, $N$ is the number of sub-carriers, $E\{\cdot\}$ is the expected value and $E\left\{|x(t)|^{2}\right\}$ is the average power of an OFDM symbol.

An OFDM symbol is formed by the superposition of many sinusoid signals of different orthogonal frequencies. Some of these subcarriers can add up constructively resulting in a peak, whose instantaneous power is much larger than the average power of the OFDM symbol. Statistically, it is possible to characterize the PAPR distribution (probability that PAPR exceeds given threshold $\gamma$ ) using complementary cumulative distribution function (CCDF). Indeed, the (CCDF) function is often used to characterize the probability that PAPR of an OFDM signal exceeds a given threshold $\left(\mathrm{PAPR}_{0}\right)$, which can be expressed as:

$$
C C D F=\operatorname{Pr}\left(P A P R>P A P R_{0}\right)
$$

Since most practical systems deal with discrete-time signals, the maximum amplitude of $L N$ samples of $x(t)$ is reduced. Parameter $L$ denotes the oversampling factor. The case of $L=1$ is 
known as critical sampling or Nyquist rate sampling, whereas values of $L>1$ correspond to oversampling. It has been proved that $L=4$ is sufficient for capturing the continuous-time peaks [20]. In this case, the discrete-time signal can be written as:

$$
x_{L}=Q_{L} X_{L}
$$

Where $Q_{L}$ are the IDFT matrix of size $N L$ scaled by $\sqrt{L}$ and $X_{L}$ the zeros-padding of $X$ by a factor $L$. So the PAPR of the discrete-time signal $x_{L}$ is defined as:

$$
P A P R=\frac{\max _{0 \leq k<N L-1}\left|x_{k}\right|^{2}}{E\left\{\left|x_{L}\right|^{2}\right\}}
$$

To simplify the notations, we considered that $x$ and $X$ as oversampled vectors by a factor $L$.

\subsection{MIMO-OFDM System}

In all this paper, we considered a space-time block codes STBC MIMO-OFDM system (Figure.1) that employs Alamouti scheme [21], where $X=\left[\begin{array}{lllll}X_{0} X_{1} \ldots X_{\frac{N}{2}-1} & X_{\frac{N}{2}} \ldots X_{N-2} X_{N-1}\end{array}\right]$ is the data signal modulated using a quadrature amplitude modulation QAM. $X_{1}=\left[\mathrm{X}_{0}-\right.$ $\left.\mathrm{X}_{1}^{*} \ldots \mathrm{X}_{\frac{\mathrm{N}}{2}-1}-\mathrm{X}_{\frac{\mathrm{N}}{2}}^{*} \ldots \mathrm{X}_{\mathrm{N}-2}-\mathrm{X}_{\mathrm{N}-1}^{*}\right]$, and $X_{2}=\left[X_{1} X_{0}^{*} \ldots X_{\frac{N}{2}} X_{\frac{N}{2}-1}^{*} \ldots X_{N-1} X_{N-2}^{*}\right]$ are the output of Alamouti STBC.

$C$ is the additive corrective signal added in frequency domain to $X$ in order to reduce the PAPR (see Figure.2), where $C=\left[C_{0} C_{1} \ldots C_{\frac{N}{2}-1} C_{\frac{N}{2}} \ldots C_{N-2} C_{N-1}\right], C_{1}$ and $C_{2}$ are generated by the same STBC.

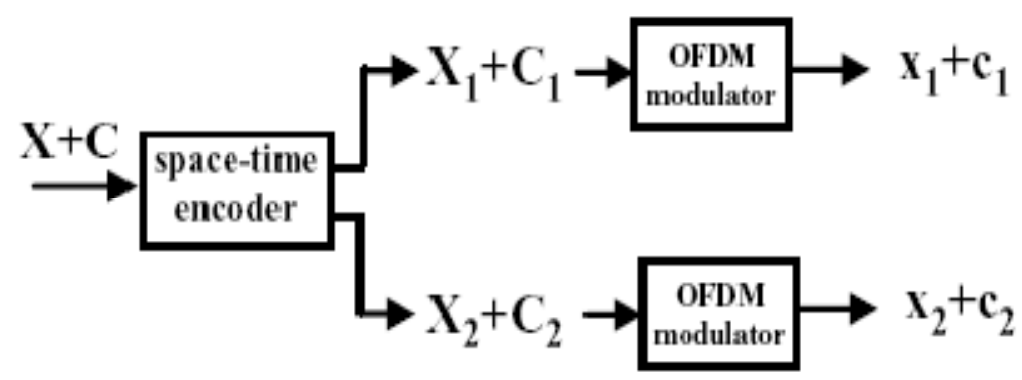

Figure 1. Structure of the two-antenna MIMO-OFDM system

To avoid non-linear distortions in power amplifiers and generation of undesired out-of-band radiation, the PAPR of all transmit signals should be simultaneously as small as possible. Since performance is governed by the worst-case PAPR, we define the PAPR for a MIMO-OFDM signal as the maximum of the PAPRs among all the parallel transmit antenna branches. PAPR at the one transmit antenna is defined as the ratio of the peak power to the average power of an OFDM signal in this branch. The MIMO-OFDM PAPR system can be expressed as [22]:

$$
P A P R_{\text {MIMO-OFDM }}=\max _{i=1, \ldots, N_{T}} P A P R_{i}
$$

Where $N_{T}$ is the number of transmission antennas. 
To evaluate the PAPR performance accurately from a statistical point of view, the complementary cumulative distribution function (CCDF) of the PAPR is used to describe the probability of exceeding a given threshold $P A P R_{0}$ defined as (3).

Our proposed strategy for PAPR reduction is to search an additive corrective signal $c$ in order to have PAPR $(\mathrm{x}+\mathrm{c})<\operatorname{PAPR}(x)$. This approach belongs to the additive signal methods for PAPR reduction. One way to find $c$ is to use optimization approaches, what is detailed in the next section.

\subsection{Proposed Approach}

The effectiveness of our proposed method is evaluated for WiMax systems. Thus, the performance evaluation is conducted in terms of PAPR reduction on WiMax systems based on the OFDM mode of the IEEE 802.16d specifications.

In this paper, we used 2x2 MIMO-OFDM as an example to introduce our idea but the proposed approach is not limited to the $2 \times 2$ system, and it can be generalized to any other similar systems $\left(\mathrm{N}_{\mathrm{Tx}}, \mathrm{N}_{\mathrm{Rx}}\right.$ with $\mathrm{T}_{\mathrm{x}}$ and $\mathrm{R}_{\mathrm{x}}>2$ ).

In fact, the strategy used is to add an artificial signal to unused carriers. The signal addition is achieved in frequency domain as presented in [17] and illustrated in Figure.2. Thus, the PAPR of the resulting signal $(x+c)$ is given by:

$$
\operatorname{PAPR}=\frac{\max _{0 \leq k<N L-1}\left|\mathrm{x}_{\mathrm{k}}+\mathrm{c}_{\mathrm{k}}\right|^{2}}{\mathrm{E}\left[|\mathrm{x}+\mathrm{c}|^{2}\right]}
$$

In reality, the ideal method to reduce PAPR would be to minimize the maximum peak of the combining signal $\left(x+c=x+Q_{L} C\right)$ while keeping constant the average power. This objective can be formulated mathematically by:

$$
\min _{\mathrm{C}} \max _{\mathrm{k}}\left|\mathrm{x}_{\mathrm{k}}+\mathrm{q}_{\mathrm{k}, \mathrm{L}}^{\mathrm{row}} \mathrm{C}\right|
$$

Where $q_{k, L}^{\text {row }}$ is the $\mathrm{k}^{\text {th }}$ row of $Q_{L}$.

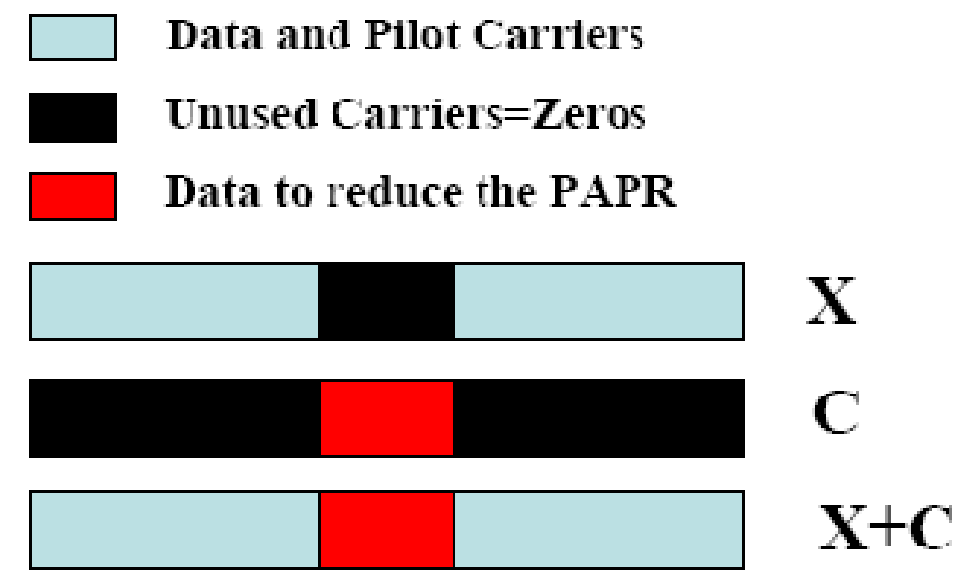

Figure 2. Example of WiMax IEEE 802.16 standard, 256 carriers, 200 carriers are used for data (192) and pilot (8), v=56 Unused carriers

Such a problem of minimization of the maximum norm given by the preceding equation can be formulated in SOCP much easier than in semi definite program (SDP) or quadratically constrained quadratic program (QCQP) [23]. 
Indeed, SOCP is a convex optimization problem class that minimizes a linear function over the intersection of an affine set and the product of second-order (quadratic) cones [23]. Many free software solvers exist to solve the convex optimization problem among which SeDuMi, YALMIP, and CVX. For our simulations, we have chosen YALMIP.

\subsection{PAPR Reducing in MIMO-OFDM Systems}

\subsubsection{Description of Tone Reservation Method for SISO-OFDM}

Tone reservation (TR) is an efficient technique to reduce the PAPR of a multicarrier signal. This method is based on adding a data-block-dependent time domain signal to the original multicarrier signal to reduce its peaks. This time domain signal can be easily computed at the transmitter and stripped off at the receiver.

For this technique, the transmitter does not send data on a small subset of subcarriers that are optimized for PAPR reduction [24]. The objective is to find the time domain signal to be added to the original time domain signal $\mathrm{x}$ such that the PAPR is reduced. If we add a frequency domain vector $C=\left[C_{0}, C_{1}, \ldots, C_{N-1}\right]^{T}$ to $\mathrm{X}$, the new time domain signal can be represented as $x+c=I D F T\{X+C\}$, where $\mathrm{c}$ is the time domain signal due to $\mathrm{C}$. The TR technique restricts the data block $X$ and peak reduction vector $\mathrm{C}$ to lie in disjoint frequency subspaces (i.e., $X_{n}=0, n \in\left\{i_{1}, i_{2}, \ldots, i_{v}\right\}$ and $C_{n}=0, n \in\left\{i_{1}, i_{2}, \ldots, i_{v}\right\}$ ). the $v$ nonzero positions in $\mathrm{C}$ are called peak reduction carriers (PRCs). Since the subcarriers are orthogonal, these additional signals cause no distortion on the data bearing subcarriers. To find the value of $C_{n}, n \in$ $\left\{i_{1}, i_{2}, \ldots, i_{v}\right\}$, we must solve a convex optimization problem that can easily be cast as a linear programming (LP) problem. To reduce the computational complexity of LP, a simple gradient algorithm is also proposed in [24] and another one proposed in [17], which is extended in this paper to the MIMO case.

\subsubsection{Tone Reservation Method via SOCP Formulation for MIMO-OFDM}

In the time-domain, each branch of the MIMO-OFDM system (figure 1) provides an output signal OFDM expressed as:

$$
\begin{aligned}
& \bar{X}_{1}=x_{1}+c_{1} \\
& =x_{1}+Q_{L}\left(\begin{array}{lll}
C & A_{1}-C^{*} & A_{2}
\end{array}\right) \\
& \bar{X}_{2}=x_{2}+c_{2} \\
& =x_{2}+Q^{\prime}{ }_{L}\left(C^{*} A_{1}+C A_{2}\right)
\end{aligned}
$$

Where $(.)^{*}$ denotes the complex conjugate operation, denotes the element-wise (dot) product of two same-size vectors, $Q^{\prime}{ }_{L}$ is the matrix from the Fourier matrix $Q_{L}$ by the permutation of the pair and odd lines of $Q_{L}$ (see Appendix), $A_{1}=\left[\begin{array}{lllllll}1 & 0 & 1 & 0 & \ldots & 1 & 0\end{array}\right]^{T}$ and $A_{2}=\left[\begin{array}{lllllll}0 & 1 & 0 & 1 & \ldots & 10\end{array}\right]^{T}$ are $N L \times 1$ vectors where $T$ denotes the transpose of the vector. The PAPRs of the signals to be transmitted are written now:

$$
\begin{gathered}
P A P R_{1}=\frac{\max _{\mathrm{k}}\left|\mathrm{x}_{1 \mathrm{k}}+\mathrm{c}_{1 \mathrm{k}}\right|^{2}}{\mathrm{E}\left\{\left|\mathrm{x}_{1 \mathrm{k}}+\mathrm{c}_{1 \mathrm{k}}\right|^{2}\right\}} \\
P A P R_{2}=\frac{\max _{k}\left|x_{2 k}+c_{2 k}\right|^{2}}{E\left\{\left|x_{2 k}+c_{2 k}\right|^{2}\right\}}
\end{gathered}
$$


According to equation (6), the PAPR of MIMO-OFDM system is:

$$
P A P R_{M I M O-O F D M}=\max \left\{P A P R_{1}, P A P R_{2}\right\}
$$

The objective of this method is to reduce the $P A P R_{M I M O-O F D M}$ what mean reducing the $P A P R_{1}$ and $P A P R_{2}$ in a joint way. In a first time, our SOCP algorithm does not take into account the increase of mean power problem.

So, the problem of PAPR minimization is equivalent then to the relations:

$$
\begin{gathered}
\min _{c_{1}} \max _{k}\left|x_{1 k}+c_{1 k}\right|^{2} \\
=\min _{c 1}\left\|x_{1}+c_{1}\right\|=\min _{c}\left\|x_{1}+Q_{L}\left(\begin{array}{ll}
C & A_{1}-C^{*} A_{2}
\end{array}\right)\right\| \\
=\min _{c 2}\left\|x_{2}+c_{2}\right\|=\min _{c}\left\|x_{2}+Q_{L}^{\prime}\left(C^{*} A_{1}+C A_{2}\right)\right\|
\end{gathered}
$$

Equations (14) and (15) convex optimization problems are equivalent to one problem which can be formulated as a SOCP:

$$
\begin{array}{cl}
\text { Minimize } & \beta \\
\text { subject to } & \left\|x_{1}+Q_{L}\left(C \square A_{1}-C^{*} \square A_{2}\right)\right\| \leq \beta \\
& \left\|x_{2}+Q_{L}^{\prime}\left(C^{*} \square A_{1}+C \square A_{2}\right)\right\| \leq \beta
\end{array}
$$

\section{SIMULATION RESULTS}

In order to validate our proposed PAPR reduction method, our results are compared with previously works [18]. In fact, the IDFT/FFT size of the WiMax signal was 256 and 56 unused tones were employed. Indeed, in the works [18], a PAPR reduction has been obtained about 2.5 $\mathrm{dB}$ at the $10^{-3}$ probability level achieved for the both antennas respectively.

Our proposed approach, based on SOCP with unused tones as Reserved Tones without constraints, will improve this reduction about $5 \mathrm{~dB}$ at the $10^{-3}$ probability level. In addition, and precisely when using CCDF of signal technique optimized with constraint mean power vs. $\gamma$, the PAPR was reduced about $6 \mathrm{~dB}$ at the $10^{-3}$ probability level.

Figure 3 shows the CCDF of the PAPR of original and optimized signals for randomly generated QPSK symbols. MIMO-OFDM system uses two antennas with $N=256$ carriers per antenna, among those, 56 unused free carriers (as in IEEE 802.16 d/e WiMax standard) which are used to add the corrective signal. We settled the oversampling factor $L=4$. In fact, to evaluate the PAPR reduction performance, we concentrated on the complementary cumulative distribution function: this is why Figure 3 compares the PAPR CCDF of the original signal with that of reduced PAPR signal without any constraint.

To solve the convex optimization problem, a free software known as YALMIP is used. As we can see, the PAPR is reduced about $5 \mathrm{~dB}$ at the $10^{-3}$ probability level.

However, adding a signal $c$ to signal $x$ to reduce the PAPR increases the transmit power. The relative increase mean power $\Delta E$, due to PAPR reduction is defined as [17]:

$$
\Delta E=10 \log _{10} \frac{E\left(|x+c|^{2}\right)}{E\left(|x|^{2}\right)}
$$


This parameter must be as small as possible in order to be compatible with current power amplifiers and to avoid power amplification saturation.

Indeed, it is clear that when the average power of the signal $x+c$ (numerator of eq.17) increases indefinitely with a constant denominator, we would have obviously a resulting PAPR of $0 \mathrm{~dB}$ related to a signal which cannot be transmitted.

Thus, the relative mean power must be upper bounded and can be written as:

$$
\Delta E \leq \gamma d B
$$

That is

$$
E\left(|x+c|^{2}\right) \leq \lambda E\left(|x|^{2}\right)
$$

where $\lambda=10^{\frac{\gamma}{10}} . \quad \gamma$ is a constant closely related to the characteristic of the power amplifier. The above condition can be added as a constraint in the optimization problem.

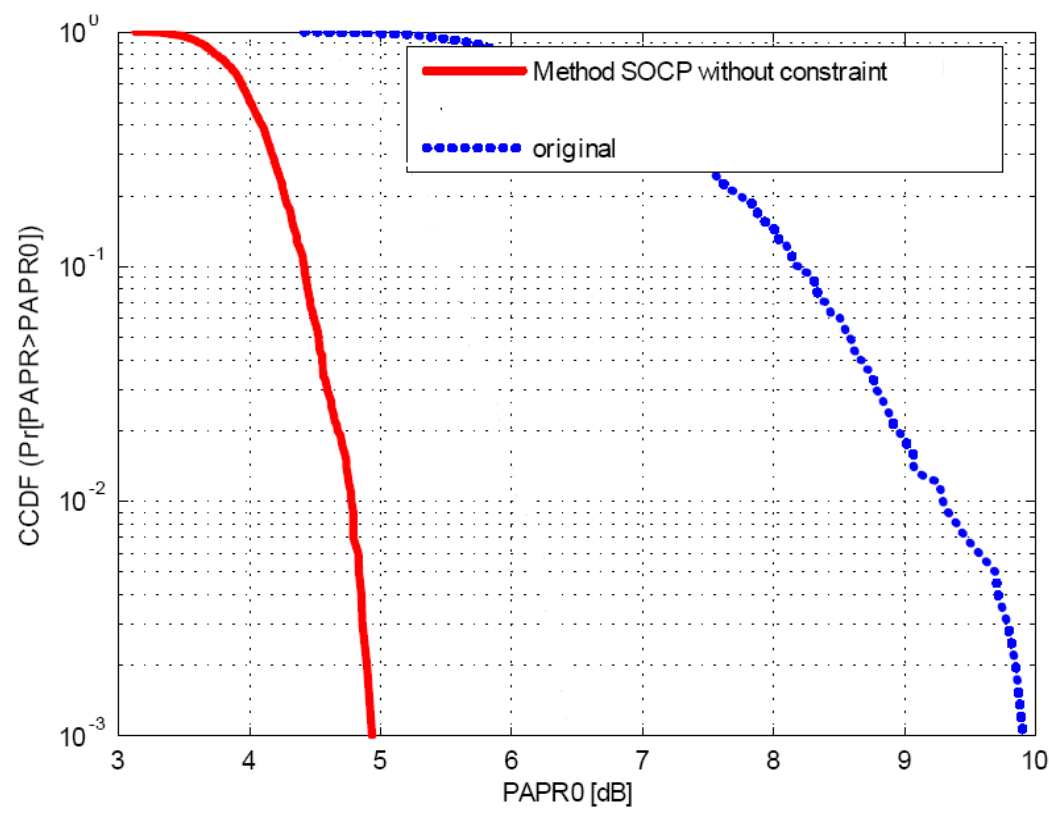

Figure 3. CCDF performance of method using SOCP with unused tones as Reserved Tones

Figure 4 plots the CCDF of the optimized signal for several $\gamma$ values. As we can see, the PAPR decreases as $\gamma$ increases. In fact, we have shown that the performances given by our approach, the PAPR can be reduced about $6 \mathrm{~dB}$ at the $10^{-3}$ probability level.

\section{Conclusions}

In this paper, we have been presented the significant improvements approved to MIMO-OFDM system based on the Tone Reservation method. We initially reformulated TR method in the form of a SOCP and taking into account the increase in the transmitted power generated by 
modelling SOCP, we added an additional constraint to control the increase in the transmitted power. Indeed, we have proposed a novel PAPR reduction approach for MIMO-OFDM based on SOCP. The proposed method is relatively simple, robust and precise and it achieved significant reduction in PAPR without transmitting any side information and without degrading BER and data rate at the same time.

To do so, we have used the unused tones of the standards to generate the corrective signal. We have shown that the performances given by our approach, the PAPR can be reduced about $6 \mathrm{~dB}$ at the $10^{-3}$ probability level. To avoid an increase of the relative mean power, we have added an additional constraint and the results still show significant gains on the CCDF of the PAPR.

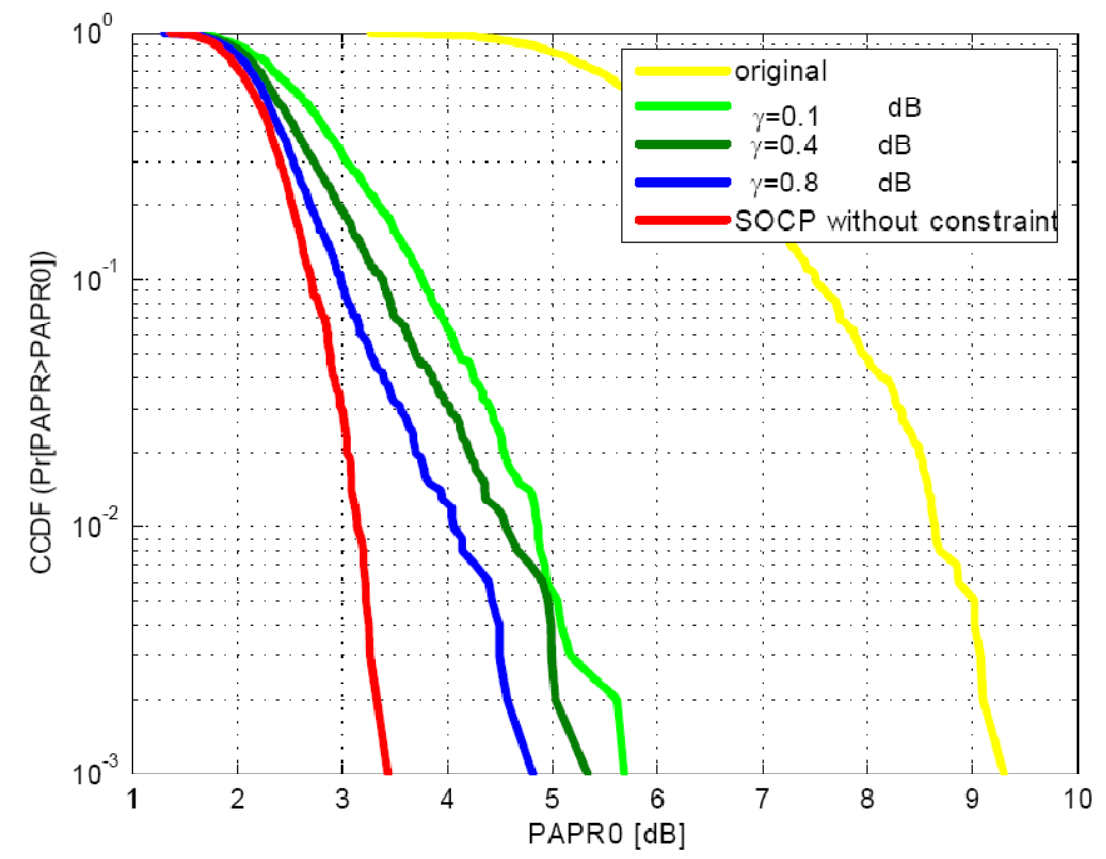

Figure 4. Performance in terms CCDF of signal optimized with constraint mean power vs. $\gamma$.

\section{APPENDIX}

$$
\begin{aligned}
& Q_{L}=\frac{1}{\sqrt{L N}}\left[\begin{array}{cccc}
1 & 1 & \cdots & 1 \\
1 & e^{j \frac{2 \pi}{L N} 1.1} & \cdots & e^{j \frac{2 \pi}{L N} 1 .(L N-1)} \\
\vdots & \vdots & \ddots & \vdots \\
1 & e^{j \frac{2 \pi}{L N}(L N-2) .1} & \cdots & e^{j \frac{(L N-2) \pi}{L N}(L N-1)} \\
1 & e^{j \frac{2 \pi}{L N}(L N-1) .1} & \cdots & e^{j \frac{(L N-1) \pi}{L N}(L N-1)}
\end{array}\right]
\end{aligned}
$$

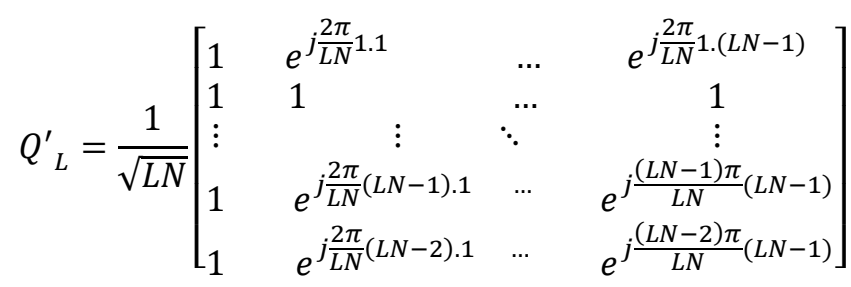




\section{REFERENCES}

[1] I. E. Telatar, (1995), "Capacity of multi-antenna Gaussian channels", ATT Bell Labs Internal Tech.

[2] G. J. Foschini \& M. J. Gans, (1998), ”On limits of wireless communications in a fading Environment when using multiple antennas", Wireless Personal Communication, vol. 6, pp: 314-335.

[3] A .José, M.R. Cantero, M.Julia, F-G.Garcia, (2006),"A MIMO-OFDM based system with backward compatibility with IEEE802.16", European cooperation in the field of scientific and technical research, 10 $0^{\text {th }}$ MCM cost 289, 23-24 March, Serbia and Montenegro.

[4] V. P. G. Jiménez, M. J. Fernández-G. Garcia, M. P. Sánchez-Fernández, A.G. Armada, (2008), " Efficient Implementation of Complementary Golay Sequences for PAP Reduction and Forward Error Correction in OFDM-based WLAN systems", AEUE-International Journal of Electronics and Communications, vol. 62, No. 9, pp: 683-694.

[5] S. H. Han \& J. H. Lee, (2005), ”An overview of peak-to-average power ratio reduction techniques for multicarrier transmission”, IEEE Wireless Communication, vol. 12, No. 2, pp: 56-65.

[6] J. Jang \& K. B. Lee, (2003), “Transmit Power Adaptation for Multi-user OFDM Systems”, IEEE Journal on Selected Areas in Communications, vol. 21, No. 2, pp: 171-178.

[7] W. Rhee \& J. M. Cioffi, (2000), "Increasing in Capacity of Multi-user OFDM System Using Dynamic Sub-channel Allocation”, in Proc. IEEE Int. Vehicular Tech. Conf., VTC'00, vol. 2, pp: 1085-1089, Springer. ISBN:0-7803-5718-3/00/\$10.00.

[8] I. Kim, H. L. Lee, B. Kim, \& Y. H. Lee, (2001), "On the Use of Linear Programming for Dynamic Sub Channel and Bit Allocation in Multi-user OFDM", in Proc. IEEE Global Communications Conf., vol. 6, pp: 3648-3652. ISBN:0-7803-7206-9/01/\$17.00.

[9] T.S.Rapport, (2002), "Wireless Communications: Principles and Practice”, Prentice Hall PTR.

[10] J. G. Proakis, (2000),” Digital Communications”, McGraw Hill, New York, $4^{\text {th }}$ edition.

[11] T.C.W.Schenk, P.F.M.Smulders \& E.R.Fledderus, (2005), "Peak-to-average power reduction in Space division multiplexing based OFDM system through spatial shifting”, Electronics Letters, Vol. 41, No. 15, $21^{\text {st }}$ July. Online N: 20051677. DOI: 10.1049/el: 20051677.

[12] Y. Lee, Y. You, W. Jeon, J. Paik, \& H. Song, (2003), "Peak-to-average power ratio in MIMOOFDM systems using selective mapping”, IEEE Communications Letters, vol. 7, No. 12, pp: 575-577.

[13] M. Tan, Z. Latinovic, \& Y. Bar-Ness, (2005), "STBC MIMO-OFDM Peak-to-Average Power Ratio Reduction by Cross-Antenna Rotation and Inversion", IEEE Communications Letters, vol. 9, No. 7, pp: 592-594.

[14] H. Lee, D. N. Liu, W. Zhu, \& M. P. Fitz, (2005), "Peak power reduction using a unitary rotation in multiple transmit antennas", in Proc. IEEE International Conference on Communication, Seoul, 
International Journal of Distributed and Parallel Systems (IJDPS) Vol.2, No.3, May 2011

South Korea, vol. 4, pp: 2407-2411. ISBN:0-7803-8938-7/05/\$20.00.

[15] Z. Latinovic, \& Y. Bar-Ness, (2006), "SFBC MIMO-OFDM Peak-to-Average Power Ratio Reduction by Polyphase Interleaving and Inversion", IEEE Communications Letters, vol. 10, No.4, pp: 266-268.

[16] A. Aggarwal, E.R. Sauffr, T.H. Meng,(2006), ”Optimal Peak-to-Average Power Ratio Reduction in MIMO Systems", IEEE International Conference on Communication ICC'06, Istanbul, Turkey, vol. 7, pp: 3094-3099.

[17] S. Zabre, J. Palicot, Y. Louet, \& C. Lereau, (2006), "SOCP approach for OFDM peak-to-average power ratio reduction in the signal adding context", IEEE ISSPIT' 06, Vancouver, Canada, August 27-30, pp: 834-839. ISBN:0-7803-9753-3.

[18] I. Braz, L. Guan, A. Zhu \& TJ. Brazil, (2010), "Peak-to-average power ratio reduction of SFBC MIMO-OFDM signals using unused tones”, DOI: 978-1-4244-5753-3/10/2010 IEEE.

[19] A. G. Armada , (2010), "SNR degradation in MIMO-OFDM systems with channel estimation errors and partial phase noise compensation", IEEE Transactions on Communication, vol. 58, No. 8, DOI: 10.1109/TCOMM.2010.070610.090159.

[20] G. Wunder and H. Boche, (2003), "Peak value estimation of band limited signals from their samples, noise enhancement, and a local characterization in the neighborhood of an extremum", IEEE Trans. Signal Processing, vol.51, n³, pp: 771-780.

[21] S. M. Alamouti, (1998), "A simple Transmit Diversity Technique for Wireless Communications", IEEE Journal on Select Areas in Communications, vol. 16, No. 8, pp: 1451-1458.

[22] R.F.H. Fischer, M. Hoch, (2007), "Peak-to-Average Power Ratio Reduction in MIMO OFDM", IEEE International Conference on Communications (ICC 2007), United Kingdom, pp: 762-767. ISBN:1-4244-0353-7/07/\$25.00.

[23] M. Lobo, L. Vandenberghe, S. Boyd, \& H. Lebret, (1998), "Applications of second order cone programming", Linear Algebra and its Applications, Special Issue on Linear Algebra in Control, Signals and Image Processing, 284(193-228).

[24] J. Tellado-Mourelo, (1999), "Peak to Average Power Ratio Reduction for multicarrier modulation", Ph.D thesis, Stanford University. 
International Journal of Distributed and Parallel Systems (IJDPS) Vol.2, No.3, May 2011

\section{Authors}

\section{PR. MAHMOUD ABDELLAOUI}

Received the B.S. degree in Electronic Engineering from High National Engineering School of Electronics and their Applications (ENSEA), Cergy, France, in 1988, and the M.S. degree from Lille University, France, in 1989. He received his Ph.D. degree in Electronic Engineering from Ecole Centrale de Paris, in 1991. He received his Habilitation degree in Electrical Engineering from National Engineering School of Sfax, Tunisia, in 2007. From October 1991 to September 1995, he was a Post doctor with Electronics \& Telecommunications Research group in ENSEA-Cergy University, France. From September 1996, he has been an Assistant Professor at INSAT \& ISECS, Tunisia. In October 2007, he was promoted to the rank of Associate Professor at High National Institute of Electronics and Communications, Sfax, Tunisia. His current research interests include wireless communications like Link and System capacity, Multi-standards MIMO-OFDM systems; Mobile Networking (mobile IP networks, VoIP, networking wireless networks based on ISPDPLL; Wireless Sensor Network (WSN). He's the author of many published articles in: International Journal on Wireless Personal Communication (Springer); International Journal of Computer Sciences; American Journal of Engineering and Applied Sciences; International Journal of Electronics \& Communications (AEÜ)-ELSEVIER; IEE Electronics letters; IEEE-MTT, etc. Dr. Abdellaoui has been invited as a reviewer of IEEE communications letters, International Journal of Electronics and Communications (ELSEVIER), Wireless Personal Communication (Springer), etc.

\section{EMNA HAJLAOUI}

Received the B.S. degree in 2005 from National Engineering School of Gabes, Tunisia, and M.S. degree in 2007 from High Engineering School of Telecommunications (SUP'COM), Tunis, Tunisia. Her Research interests focus on multi-standards MIMO-OFDM systems, MIMO-max- $\mathrm{d}_{\text {min }}$ Criterion, PAPR reduced for MIMO-OFDM systems. 